\title{
Beschäftigung zwischen Mobilität und Stabilität: Empirische Befunde und wirtschaftspolitische Folgerungen
}

\author{
Knut Gerlach • Olaf Hübler • Gesine Stephan
}

Angenommen: 25. Januar 2011 / Online publiziert: 10. Februar 2011

(C) Institut für Arbeitsmarkt- und Berufsforschung 2011

Zusammenfassung Ziel dieses Beitrags ist, einen Überblick über unsere neueren Forschungsergebnisse zum Spannungsfeld der Beschäftigung zwischen Mobilität und Stabilität - aus individueller und betrieblicher Sicht sowie unter Berücksichtigung institutioneller Einflüsse - zu geben. Die vorgestellten Arbeiten gehen stärker als die bisherige Literatur auf die Interaktionen zwischen Individuum und Betrieb ein. Die Ergebnisse lassen sich vier Projektschwerpunkten zuordnen. Erstens identifizierte eine Anzahl von Studien individuelle, betriebliche und gesamtwirtschaftliche Determinanten der Beschäftigungsstabilität. Zweitens wurde die Bedeutung von Löhnen und Zufriedenheit auf Arbeitsplatzwechsel untersucht. Die Befunde zeigen unter anderem, dass höhere Arbeitsplatzsicherheit und höhere Löhne in Deutschland Hand in Hand gehen; allerdings lassen sich auch Hinweise auf kompensierende Lohndifferentiale finden. Ein dritter Schwerpunkt analysiert die Bedeutung von Institutionen für die Beschäftigungsstabilität. Die Ergebnisse weisen darauf hin, dass die Beschäftigung in Betrieben, die Tarifverträge anwenden, stabiler ausfällt, während betriebliche Bündnisse zumindest mittelfristig keine positiven Beschäftigungswirkungen hatten. Schließlich ging ein vierter Projektteil der Wirkung von Eingliederungszuschüssen auf Löhne und Beschäftigungsdauer nach. Dabei

K. Gerlach · O. Hübler ( $\varangle)$

Leibniz Universität Hannover, Königsworther Platz 1, 30167 Hannover, Deutschland

e-mail: huebler@ewifo.uni-hannover.de

\section{G. Stephan}

Institut für Arbeitsmarkt- und Berufsforschung (IAB),

Regensburger str. 104, 90478 Nürnberg, Deutschland

G. Stephan

Universität Erlangen-Nürnberg, Nürnberg, Deutschland zeigte sich, dass geförderte Beschäftigungsverhältnisse bei ungefähr gleich hohen Einstiegslöhnen länger andauern.

Schlüsselwörter Beschäftigungsstabilität .

Betriebszugehörigkeitsdauer · Arbeitsplatzwechsel, Löhne · Arbeitszufriedenheit · Tarifverträge · Öffnungsklauseln ·

Eingliederungszuschüsse

JEL Klassifikationen J28 · J31 · J62 · J68 · J5

\section{Employment stability and mobility: empirical findings} and policy conclusions

Abstract The objective of this paper is to present a short survey of our new research results to mobility and stability of employment on a micro level. This area of conflict is analyzed from an individual and a firm's perspective, taking institutional issues into account. More than previous studies, we investigate interactions between individual and firm side issues. The results can be assigned to four subsections. First, individual, firm and macroeconomic determinants of employment stability are identified. Second, the relevance of wages and job satisfaction for job changes is investigated. In addition to other findings, the results show for Germany that high job stability and high wages go hand in hand. There exist also unobserved determinants which operate as compensating wage differentials. Third, the impact of institutions on job mobility is analysed. The outcome demonstrates that employment in firms with a collective agreement is more stable than in other firms, while company level pacts are not very successful in stabilizing employment at least in the medium term. Fourth, the effects of wage subsidies on wages and employment duration are investigated. The analysis shows that subsidised workers stay significantly longer at 
their first employer than similar unsubsidised workers, while entry wages do not differ significantly between both groups.

\section{Einleitung}

Beschäftigungsbewegungen auf dem Arbeitsmarkt sind immer Gegenstand theoretischer, empirischer und politischer Diskussionen. Mal wird auf gesamtwirtschaftlicher Ebene eine zu geringe Beweglichkeit, zu starke Inflexibilität beklagt, mal wünscht man sich mehr Stabilität. Die Einschätzungen über den richtigen Zeitpunkt eines Arbeitsplatzwechsels schwanken ebenso wie über das wünschenswerte Ausmaß eines Austauschs von Arbeitskräften durch die Unternehmen. Die wirtschaftspolitischen Akteure versuchen, ungünstigen Entwicklungen durch ein breites Spektrum an Maßnahmen entgegen zu wirken. Das von der DFG von 2004-2010 im Rahmen des Schwerpunktprogramms ,Flexibilisierungspotenziale bei heterogenen Arbeitsmärkten“ geförderte Forschungsprojekt ,Beschäftigung zwischen Mobilität und Stabilität" fällt insofern in eine interessante Phase wirtschaftlicher Entwicklung, als zu Beginn des Projekts in der wissenschaftlichen und öffentlichen Diskussion Forderungen nach mehr Flexibilität dominierten, während in den Jahren 2008 und 2009 von verschiedenen Seiten der Ruf nach mehr Stabilität laut wurde. Zentrales Ziel der Forschungsarbeiten ist es, herauszuarbeiten, was die Entscheidungen der verschiedenen Wirtschaftsakteure bestimmt, einen Arbeitsplatz beizubehalten oder zu wechseln, Arbeitskräfte auszutauschen, betriebsintern umzusetzen oder zu befördern. Das Projekt untersucht weiterhin, welche Rolle Arbeitsmarktinstitutionen für die Wirksamkeit unternehmens- und wirtschaftspolitischer Maßnahmen spielen. Hierzu werden verschiedene Mikrodatensätze und ökonometrische Methoden eingesetzt. Dieser Beitrag stellt die wesentlichen Befunde des Projektes vor und fasst zentrale wirtschaftspolitische Folgerungen zusammen.

Farber (1999) gab am Übergang zum neuen Jahrtausend einen Überblick zum Stand der empirischen Mobilitätsforschung auf dem Arbeitsmarkt. Seine Analyse verdeutlicht, dass Individual- und Unternehmenspaneldaten erforderlich sind, um zentrale Determinanten von Mobilität und Stabilität herauszuarbeiten. Neben zwischenbetrieblicher ist auch die innerbetriebliche Mobilität zu untersuchen. Spezifisches Humankapital sowie die Heterogenität zwischen Arbeitnehmergruppen sind wichtige Determinanten von Arbeitskräftemobilität und Lohndynamik. Seitdem ist nicht nur in unserem Projekt über die Thematik „Mobilität versus Stabilität von Beschäftigung" geforscht worden. Eine Reihe neuer Erkenntnisse hat zu einem besseren Verständnis beigetragen, warum Mobilität nicht zu jedem Zeitpunkt gleichermaßen ausgeprägt ist, und warum einzelne Arbeitnehmergruppen stärker zu Arbeitsplatzwechseln neigen als andere. Daraus ergibt sich, dass stabilisierende oder flexibilisierende wirtschaftspolitische Maßnahmen nicht immer und gleich wirksam sind.

Für die Lebensplanung von Arbeitnehmern ist ein gesicherter Arbeitsplatz wichtig. Das heißt nicht zwangsläufig, dass sie lebenslang beim gleichen Arbeitgeber bleiben wollen. Motive wie eine Verbesserung der Einkommens- oder Arbeitssituation wie auch eine bessere Abstimmung zwischen privaten Interessen und Erwerbstätigkeit können Anlass sein, sich nach einem neuen Arbeitsplatz umzusehen. Ob diese Suche in einem Arbeitsplatzwechsel mündet, wird durch die Ansprüche und Alternativen determiniert, die sich zwischen Personengruppen stark unterscheiden. Nicht zuletzt hängen die Möglichkeiten eines Arbeitsplatzwechsels oder einer Beschäftigungsaufnahme von den Zielen potenzieller neuer Arbeitgeber ab. Auf der einen Seite kennen Unternehmen die speziellen Fähigkeiten ihrer Arbeiter erst nach einer gewissen Zeit gut und können sie dann bestmöglich einsetzen. Nur bei einer längerfristigen Beschäftigung kann auch ein hinreichender Nutzen aus zielgenauen Weiterbildungsaktivitäten gezogen werden. Auf der anderen Seite können veränderte wirtschaftliche Rahmenbedingungen Anpassungen bei der Beschäftigung erfordern, für die interne Maßnahmen - wie Umsetzungen, betriebsorganisatorische Änderungen und Qualifizierung - nicht ausreichen. Entlassungen und der Austausch eines Teils der Belegschaft mit einem anderen Qualifikationsprofil sind unvermeidliche Konsequenzen. Erst die Verfügbarkeit neuer Datenquellen hat es in den letzten Jahren ermöglicht, einige zum Teil bereits lange theoretisch diskutierte Einflüsse empirisch zu untersuchen.

Im Folgenden skizzieren wir vor diesem Hintergrund eigene Befunde zu den vier Schwerpunkten des Projekts: (a) die Bestimmungsgründe der Beschäftigungsdynamik, (b) das Zusammenwirken von Löhnen, Zufriedenheit und Arbeitsplatzwechsel, (c) die Bedeutung von Institutionen für die Stabilität der Beschäftigung sowie (d) die Stabilität und Entlohnung bei geförderter Beschäftigung. Unsere Untersuchungen machen deutlich, dass fast immer mehrere (und nicht nur ökonomische) Dimensionen die Entscheidung für stabile Beschäftigung oder aber Mobilität beeinflussen. Tabelle 1 stellt im Überblick dar, welche Datensätze den erörterten Studien zugrundeliegen, welche Methoden eingesetzt wurden, und welches die zentralen Ergebnisse sind.

\section{Individuelle, betriebliche und gesamtwirtschaftliche Bestimmungsgründe der Beschäftigungsdynamik}

Farber (1999) kommt in seinem Übersichtsbeitrag zu den bis dahin vorliegenden empirischen Untersuchungen zur Dichotomie ,flexible versus stabile Beschäftigung" zu dem Urteil, dass sich aus der Empirie drei stilisierte Fakten ableiten lassen: (1) Die meisten Beschäftigungsverhältnisse sind 


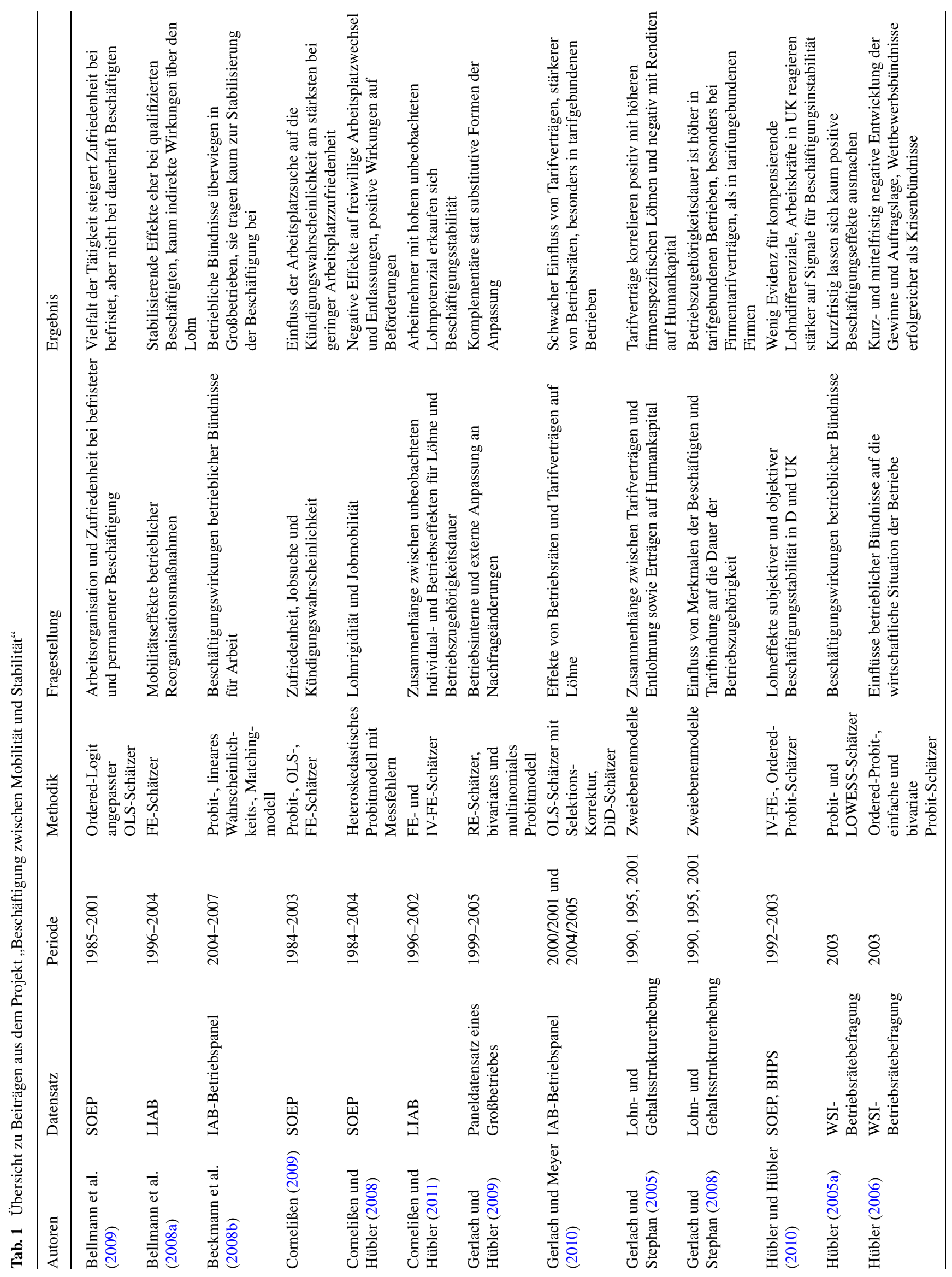




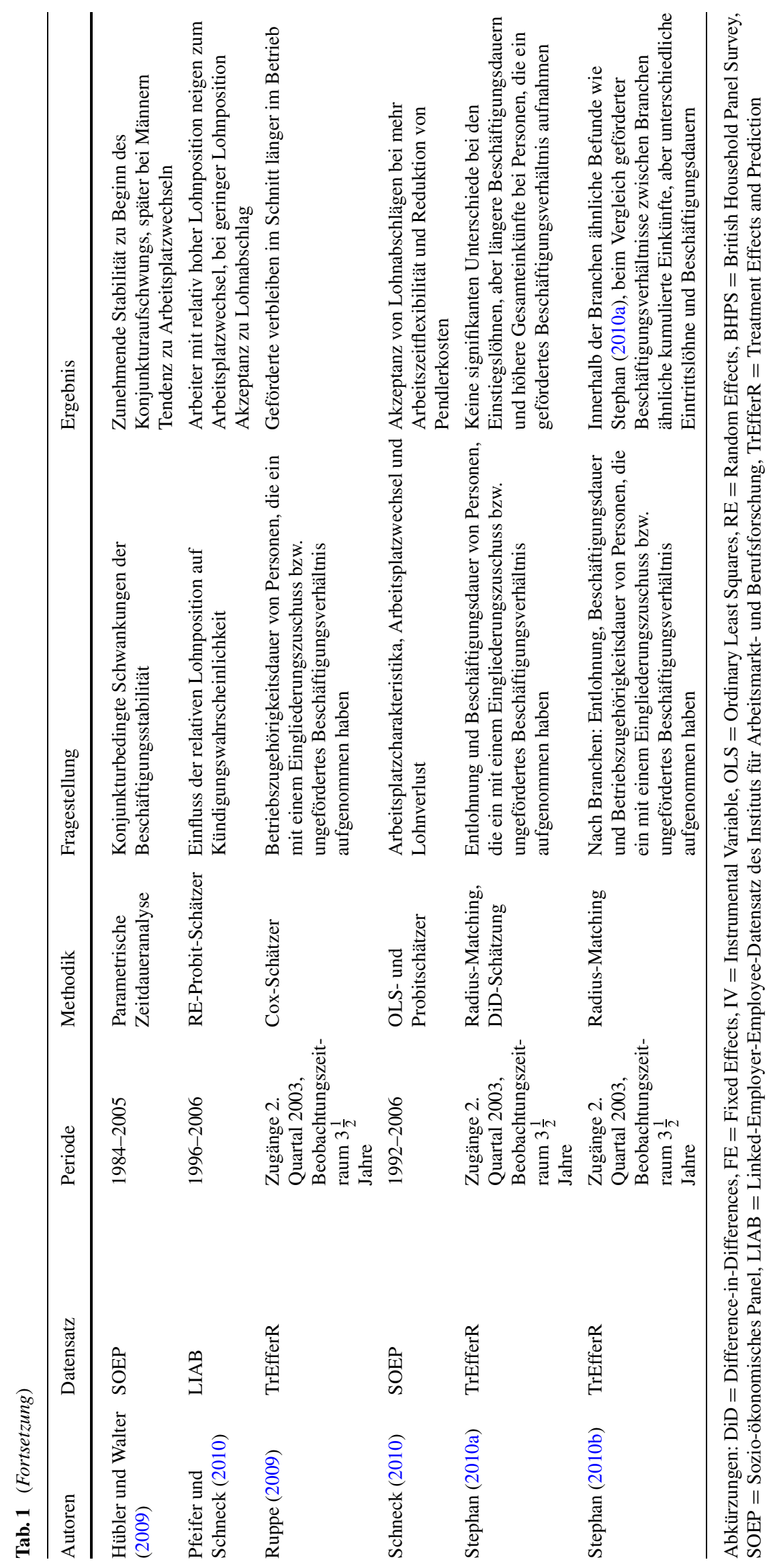


langfristig angelegt. (2) Viele Arbeitsverhältnisse dauern jedoch nicht lange an. (3) Die Wahrscheinlichkeit, dass ein Arbeitsverhältnis endet, sinkt mit der Betriebszugehörigkeitsdauer. Er zeigt weiterhin auf, dass Heterogenität und spezifisches Humankapital das Fluktuationsverhalten wesentlich determinieren. Diese Faktoren werden in empirischen Analysen im Allgemeinen jedoch eher indirekt als direkt erfasst. Es bleibt offen, ob die Bestimmungsgründe des Fluktuationsverhaltens vor allem im individuellen, im betrieblichen, im institutionellen oder im gesamtwirtschaftlichen Bereich zu finden sind. Zum individuellen Mobilitätsverhalten von Arbeitnehmern lag bereits vor mehr als einer Dekade eine Vielzahl von Befunden vor. Für die Entscheidung, ob ein Arbeitsplatz gewechselt wird oder nicht, sind jedoch nicht nur persönliche Merkmale und Präferenzen bedeutsam. Auch betriebliche Strukturen, Entwicklungen und Ziele beeinflussen den realisierten Umfang an Mobilität ganz wesentlich. Vor allem aber sind die Wechselwirkungen zwischen Arbeitnehmer- und Unternehmensverhalten von Bedeutung. Aus dieser Sicht ist unser empirisches Wissen noch ausgesprochen rudimentär.

Zwei Formen von Datensätzen, die neue Einsichten erlauben, wurden erst in den letzten Jahren verfügbar und sind in unserem Projekt genutzt worden: Dies sind erstens Paneldatensätze aus einzelnen Betriebe sowie zweitens verknüpfte Individual- und Betriebsdaten. Gerlach und Hübler (2009) nutzen einen umfangreichen Individualdatensatz der Beschäftigten eines großen deutschen Unternehmens des Verarbeitenden Gewerbes. Diese Daten haben - auch wenn Verallgemeinerungen nur beschränkt möglich sind - verschiedene Vorteile: Hoher Verlässlichkeitsgrad der Daten, keine unbeobachtbare Heterogenität in Bezug auf Unternehmens-, Wirtschaftsbereich- und Regionalmerkmale, detaillierte Angaben über Beschäftigungsanpassungen, monatliche Daten. Die Auswertungen zeigen, dass der Beschäftigungszyklus des Unternehmens zwar weitgehend, aber nicht vollständig mit den volkswirtschaftlichen Bewegungen übereinstimmt, dass interne und externe Anpassungen eher komplementär als substitutiv verlaufen und dass sich kaum analoge Lohnanpassungen ausmachen lassen. Ausgeprägte zyklische Effekte sind nicht beobachtbar. Beförderungen reduzieren die Mobilitätsneigung der Beschäftigten, Überstunden bewirken das Gegenteil.

Die Vorteile eines verknüpften Individual- und Betriebsdatensatzes liegen vor allem darin, dass gleichzeitig beobachtete und unbeobachtete Individual- und Betriebseffekte erfasst werden können. Aus dem Linked-EmployerEmployee-Datensatz des Instituts für Arbeitsmarkt- und Berufsforschung (LIAB) konnten weiterführende Befunde zur Beschäftigungsmobilität gewonnen werden. Bellmann et al. (2008a) gehen unter anderem dem Einfluss betriebsinterner organisatorischer Änderungen auf die Beschäftigungsstabilität nach. Im Ergebnis hat die Einführung von Gruppenar- beit stabilisierende Effekte auf die Beschäftigung. Demgegenüber wirken eine Verlagerung der Verantwortung und der Entscheidungsbefugnis nach unten destabilisierend. Insgesamt finden sich bei Reorganisationsmaßnahmen innerhalb der Gruppe mit mittlerem Ausbildungsniveau eher beschäftigungsstabilisierende Wirkungen als bei anderen Arbeitskräften. Insbesondere für Un- und Angelernte lässt sich ein solcher Effekt nicht feststellen. Bemerkenswert ist, dass Reorganisationsmaßnahmen entweder Lohnveränderungen zur Folge haben oder die externe Jobmobilität beeinflussen, aber seltener beides eintritt.

Neben individuellen und betrieblichen Merkmalen sind auch gesamtwirtschaftliche, insbesondere konjunkturelle Einflüsse für die Beschäftigungsmobilität bedeutsam. Hübler und Walter (2009) analysieren daher unter Verwendung der SOEP-Daten auch den Zusammenhang zwischen konjunkturellen Schwankungen und der Dauer und damit der Stabilität von Beschäftigungsverhältnissen in (West-) Deutschland. Die Ergebnisse zeigen, dass die konjunkturelle Entwicklung die Stabilität der Beschäftigung beeinflusst. Dies gilt auch, wenn für Alter, Schulbildung, Stellung im Beruf, Teilzeit, Öffentlicher Dienst, Firmengröße und Wirtschaftssektor kontrolliert wird. Das Risiko, dass ein Beschäftigungsverhältnis endet, sinkt im Konjunkturaufschwung. Es nimmt bei Männern allerdings zu, wenn das durchschnittliche Wachstum im vorletzten und vorvorletzten Jahr günstig ausfiel - hohes Wachstum in den Vorperioden erhöht offensichtlich mit Verzögerungen im Konjunkturaufschwung das Selbstkündigungsrisiko. Bei Frauen gleichen sich hingegen Selbstkündigungen und Entlassungen im Konjunkturaufschwung eher aus.

Damit ist festzuhalten: Was die Entscheidung betrifft, auf einem Arbeitsplatz zu verbleiben oder zu wechseln, wirken sich betriebliche Maßnahmen und konjunkturelle Bewegungen bei unterschiedlichen Beschäftigtengruppen nicht einheitlich aus.

\section{Arbeitsplatzwechsel, Löhne und Zufriedenheit}

Das Lohnniveau und Lohnänderungen sind aus theoretischer Sicht die wichtigsten Bestimmungsgründe für das Beschäftigungsniveau und die Beschäftigungssicherheit. In dem letzten Jahrzehnt wurde der Lohn in empirischen Studien allerdings meist nur als Kontrollvariable zur Erklärung von Arbeitsplatzwechseln herangezogen. Wir haben uns ausgewählten Fragen zum Zusammenhang zwischen Löhnen, Arbeitsplatzwechseln und Zufriedenheit zugewandt, für die bisher kaum oder keine empirischen Ergebnisse vorlagen.

Die erste dieser Fragen ist: Beeinflussen Lohnstarrheiten die interne und externe Mobilität (Cornelißen und Hübler 2008)? Lohnstarrheiten wurden dabei anhand des individuellen Grads der durch rigide Löhne hervorgerufenen 
„Lohnaufschwemmung“ bestimmt, d.h. der Differenz zwischen dem tatsächlichen Lohn und dem Wettbewerbslohn, der sich bei Abwesenheit von Lohnstarrheiten ergeben hätte. Auf der individuellen Ebene zeigt sich, dass mit steigender Lohnrigidität auch die Chancen einer betriebsinternen Beförderung zunehmen. Lohnrigidität hat aber keine Wirkungen auf innerbetriebliche Umsetzungen und hierarchische Herabstufungen. Lohnrigidität verringert gleichermaßen Kündigungen seitens der Arbeitnehmer wie auch Entlassungen durch den Arbeitgeber: Sie bedeutet für den Arbeitnehmer einen jobspezifischen Vorteil und wird daher die Neigung, den Arbeitsplatz zu wechseln, reduzieren. Wenn für einzelne Beschäftigtengruppen sowohl Entlassungen als auch stark schwankende, sich schnell den gesamtwirtschaftlichen Bedingungen anpassende Löhne zu beobachten sind und für andere das Gegenteil, dann spricht dies für die Existenz eines dualen Arbeitsmarktes. Ob dabei die Motivation des Arbeitgebers, die Kernbelegschaft zu halten, ausschlaggebend ist, oder ob die Kernbelegschaft aufgrund von Verhandlungsmacht sowohl Vorteile bei den Löhnen als auch bei der Arbeitsplatzstabilität erzielen kann, lässt sich empirisch nicht ermitteln. Diese Resultate entsprechen nur zum Teil denen von Altonji und Devereux (2000) für den amerikanischen Arbeitsmarkt, die keinen Effekt der Lohnrigidität auf Beförderungen und Entlassungen feststellen. Sie bestätigen aber für den deutschen Arbeitsmarkt Befunde von Pfeiffer (2003) zum Zusammenhang zwischen Lohnrigidität und Entlassungen.

Die zweite untersuchte Frage ist: Wie wirken sich die subjektiv empfundene und die mit objektiven Indikatoren gemessene Arbeitsplatzunsicherheit auf den individuellen Lohn aus (Hübler und Hübler 2010)? Untersuchungsergebnisse liegen für Westdeutschland und Großbritannien vor. Die Beschäftigungsstabilität wird zum einen durch regionale Arbeitslosenquoten und zum anderen durch die individuell wahrgenommene Arbeitsplatzsicherheit erfasst; zudem wird für die individuelle Dauer der Arbeitslosigkeit im vorangegangenen Jahr kontrolliert. Die Ergebnisse sprechen dafür, dass von den beiden Erklärungsansätzen - Verhandlungsmacht vs. kompensierende Lohndifferentiale - eher ersterer dominant ist. Hinweise auf die Existenz eines dualen Arbeitsmarktes sind für Großbritannien jedoch weniger ausgeprägt als für Deutschland. Interessanterweise fallen die Lohnwirkungen von Arbeitsplatzsicherheit in den beiden Ländern asymmetrisch aus. In Deutschland geht steigende Arbeitsplatzsicherheit mit höheren Löhnen einher; in England sinkende Arbeitsplatzsicherheit mit niedrigeren Löhnen. Veränderungen der Arbeitsplatzsicherheit in die jeweils andere Richtung wirken sich hingegen nicht auf die Löhne aus.

Drittens haben wir für Deutschland hinterfragt: Welche Effekte haben unbeobachtete Individualeffekte und Firmeneffekte auf Löhne und Betriebszugehörigkeitsdauer (Cornelißen und Hübler 2011)? Die Analysen bestätigen ein bisher in der Empirie als Puzzle bezeichnetes Phänomen: Arbeitskräfte mit einem hohen unbeobachteten Lohnpotential arbeiten verstärkt in Betrieben mit einem eher niedrigen Lohnniveau: Personen mit einem hohen Lohnpotenzial verzichten bei ihrer Arbeitsplatzwahl auf einen Teil des möglichen Lohns zugunsten von mehr Arbeitsplatzstabilität. Demgegenüber sind Arbeitskräfte mit einem geringen Lohnpotenzial kaum auf Arbeitsplätzen zu finden, die sich durch hohe Stabilität auszeichnen. Also gibt es doch kompensierende Lohndifferentiale? Duale Arbeitsmärkte und kompensierende Lohndifferentiale schließen sich nicht aus. Beschäftigte auf dem primären Arbeitsmarkt verfügen über Verhandlungsmacht. Sie haben die Wahl zwischen etwas mehr Sicherheit und etwas höherem Einkommen, können jedoch nicht beides gleichermaßen durchsetzen. Auf dem sekundären Arbeitsmarkt stellt sich die Situation umgekehrt dar. Die Arbeitskräfte haben keine Verhandlungsmacht. Sie können sich nur zwischen einem geringen Lohn und etwas Sicherheit oder etwas mehr Lohn und keiner Sicherheit entscheiden.

Damit könnte sich eine Erklärung für die folgende vierte Frage anbieten: Wieso gehen freiwillige Arbeitsplatzwechsel gar nicht so selten mit einem geringeren Lohn einher (Pfeifer und Schneck 2010; Schneck 2010)? Arbeitnehmer nehmen einen Lohnabschlag zugunsten von mehr Sicherheit in Kauf. Ein anderer Grund könnte eine Verbesserung der relativen Lohnposition sein. Allerdings lässt sich empirisch kein signifikanter Zusammenhang zwischen Lohnabschlag und relativer Lohnposition beobachten. Auch ist ein größeres Lohnwachstum im neuen Betrieb, verbunden mit einer anfänglichen Lohnreduktion, kaum Grund für einen freiwilligen Wechsel. Vielmehr scheinen Arbeitsbedingungen für das scheinbar irrationale Verhalten ursächlich zu sein. Dies sind insbesondere verbesserte Arbeitszeitregelungen und verminderte Kosten des Pendelns zwischen Wohnung und Arbeitsplatz.

Arbeitszufriedenheit dürfte für die Arbeitsplatzwahl entscheidender sein als der Lohn allein. Die empirischen Untersuchungen des Zusammenhangs zwischen Zufriedenheit und Mobilität (Beckmann et al. 2009; Cornelißen 2009) zeigen, dass Zufriedenheit am Arbeitsplatz insbesondere vom Verhältnis zu den Kollegen und Vorgesetzten sowie von der Sicherheit des Arbeitsplatzes und abwechslungsreichen Tätigkeiten beeinflusst wird. Geringe Arbeitsplatzzufriedenheit trägt zur verstärkten Suche nach alternativen Jobs bei; diese Suchaktivitäten haben einen signifikanten Einfluss auf tatsächliche Arbeitsplatzwechsel. Bei befristetet Beschäftigten unterscheidet sich die Zufriedenheitsreaktion in Bezug auf verschiedene betriebliche Praktiken deutlich von permanent Beschäftigten. So wirkt sich z.B. Aufgabenvielfalt positiv auf die Arbeitszufriedenheit von temporär Beschäftigten, aber weniger auf die von permanent Beschäftigten aus. Umgekehrtes scheint bei der Mitarbeiterbeteiligung zu 
gelten. Für befristet Beschäftigte spielt der Autonomiegrad bei der Arbeit kaum eine Rolle. Gute Beförderungsaussichten erhöhen die Arbeitszufriedenheit allgemein, bei temporär Beschäftigten allerdings in stärkerem Ausmaß. Bei den unbefristet Beschäftigten spielt schließlich der Lohn für die Zufriedenheit eine größere Rolle.

Insgesamt lässt sich auf Basis der Untersuchungsergebnisse festhalten, dass nicht nur eine Dimension das Verhältnis von Arbeitsplatzsicherheit, Löhnen und Zufriedenheit bestimmt: Lohnrigidität hemmt die Mobilität; gut entlohnte Arbeitskräfte haben üblicherweise auch relativ sichere Arbeitsplätze; freiwillige Wechsel auf geringer bezahlte Arbeitsplätze lassen sich teilweise über die Theorie kompensierender Lohndifferentiale erklären.

\section{Institutionen und Stabilität der Beschäftigung}

Die Literatur zu betriebsinternen Arbeitsmärkten zeigt, dass eine längere Betriebszugehörigkeitsdauer sowohl für Arbeitnehmer als auch für Unternehmen mit ökonomischen Vorteilen verbunden ist. Bei negativen externen Nachfrageschocks und Reorganisationsmaßnahmen zur Verbesserung der betrieblichen Wettbewerbsfähigkeit ist jedoch ein ergänzendes Flexibilisierungspotenzial erforderlich, wenn langfristig stabile Beschäftigungsverhältnisse nicht gefährdet werden sollen. Dieses Potenzial umfasst Kurzarbeit, Arbeitszeitkonten, tarifvertraglich vereinbarte Arbeitszeitkorridore, Abbau von Überstunden, Weiterbildung, das Vorziehen von Aufgaben, die Kündigung von Subunternehmen und den Abbau von Randbelegschaften. In den letzten Jahren trafen Betriebe, Betriebsräte und Gewerkschaften zudem verstärkt Vereinbarungen über betriebliche Bündnisse. Institutionen wie Betriebsräte und Gewerkschaften können über Tarifverträge und Betriebsvereinbarungen kurzfristige Anpassungen des Arbeitsvolumens allerdings auch erschweren. Hinzu kommt, dass der Kündigungsschutz in Deutschland die Anpassungskosten erhöht und Entlassungen häufig langwieriger und rechtlich unkalkulierbarer macht.

In zwei Papieren untersuchen Gerlach und Stephan (2005, 2008) den Einfluss der Tarifbindung von Betrieben auf die Dauer der Betriebszugehörigkeit und die Lohnhöhe in den Jahren 1990, 1995 und 2001. Zentrale Ergebnisse sind: Die Dauer der Betriebszugehörigkeit ist in Betrieben mit einem Tarifvertrag länger als in Betrieben mit individuellen Lohnvereinbarungen. Der Effekt ist bei Firmentarifverträgen stärker ausgeprägt als bei sektoralen Lohnvereinbarungen, ein Ergebnis, dass Card und de la Rica (2006) auch für Spanien gefunden haben. Beschäftigte in Betrieben mit Tarifvertrag profitieren nicht nur von einer höheren Arbeitsplatzsicherheit, sondern erhalten auch eine höhere Entlohnung.
Die Reform des Betriebsverfassungsgesetzes im Jahr 2001 verfolgte das politische Ziel, die Bildung von Betriebsräten $\mathrm{zu}$ fördern, den gewerkschaftlichen Zugang zu Betrieben zu erleichtern und die Mitbestimmungsrechte von Betriebsräten auszuweiten. Gerlach und Meyer (2010) analysieren in Anlehnung an Hübler und Jirjahn (2003) mit dem IAB-Betriebspanel für Niedersachsen (Gerlach et al. 2003) den Lohneinfluss von Betriebsräten und der Tarifbindung von Betrieben. Ihre Untersuchung basiert auf den gepoolten Wellen der Jahre 2000/2001 und 2004/2005. Zwischen den beiden Perioden ging die Bedeutung der Betriebe mit Tarifbindung und Betriebsrat von 49 auf 39 Prozent zurück, während der Anteil der Betriebe ohne diese beiden Institutionen von 19 auf 26 Prozent zunahm. Schätzungen zum Lohneinfluss von Betriebsräten führen zu dem Ergebnis, dass dieser nur in tarifgebundenen Betrieben positiv signifikant ist und in der zweiten Periode zugenommen hat. Der Zeitraum dieser Untersuchung war geprägt von anhaltend hoher Arbeitslosigkeit und moderaten, durch die Gewerkschaften im Rahmen von Tarifverhandlungen durchgesetzten Lohnerhöhungen. Das politisch intendierte Ziel einer Förderung der Bildung von Betriebsräten wurde nicht erreicht, der Anteil von Betrieben mit Betriebsräten war deutlich rückläufig. Diese Konstellation kann dazu geführt haben, dass existierende Betriebsräte in Verbindung mit gewerkschaftlicher Unterstützung verstärkt dazu übergegangen sind, Lohnerhöhungen durchzusetzen.

Bei einem anhaltend hohem Niveau der Arbeitslosigkeit und nachlassenden Bindungswirkungen von Gewerkschaften und Arbeitgeberverbänden wurden nach der im Jahr 1984 vereinbarten Reduzierung der Arbeitszeit auf 35 Wochenstunden in der Metallindustrie nach und nach Öffnungsklauseln (Bündnisse für Arbeit) in Tarifverträge aufgenommen. Diese ermöglichen Abweichungen von tariflichen Standards zu Lasten der Belegschaft bei entsprechenden Konzessionen der Arbeitgeber, die häufig eine Beschäftigungssicherung beinhalten. Anlass für ein Bündnis für Arbeit kann eine aktuelle Krise, die Prävention gegen eine bevorstehende Krise oder die Notwendigkeit einer generellen Verbesserung der Wettbewerbsfähigkeit sein. Hübler (2005a, 2005b, 2006) nutzt bei seinen Analysen zu betrieblichen Bündnissen die Daten einer Sonderhebung der Betriebsrätebefragungen des WSI aus dem Jahr 2003. Er zeigt, dass Bündnisse vor allem dann zustande kommen, wenn Geschäftsleitung und Betriebsrat vertrauensvoll zusammen arbeiten. Abgesehen von Anfangserfolgen bauen Betriebe mit einer Beschäftigungssicherungsvereinbarung über einen längeren Zeitraum meist jedoch Beschäftigung ab. Positive Beschäftigungseffekte stellen sich erst sehr viel später ein. Differenziert man nach Maßnahmen, erweisen sich Arbeitszeitverlängerungen und Qualifikationsmaßnahmen als wesentlich effektiver als Reorganisationsmaßnahmen oder Kürzungen bei der Arbeitszeit oder beim Entgelt, um positive Beschäftigungseffekte zu erzielen. Bellmann et al. 
(2008b) greifen auf die Wellen des IAB-Betriebspanels für die Jahre 2004 bis einschließlich 2007 zurück. Dabei zeigt sich, dass betriebliche Bündnisse verstärkt in größeren Betrieben anzutreffen sind und sich durch eine längere Dauer (zwischen 2 und 5 Jahren) auszeichnen. Verfolgt wird eher das Ziel einer Verbesserung der Wettbewerbsfähigkeit. Weniger genutzt werden Bündnisse zur Bewältigung einer aktuellen oder bevorstehenden Krise. Alle vier Studien zeigen, dass Betriebe in einer wirtschaftlich ungünstigen Situation im Allgemeinen keine Beschäftigungsstabilisierung erreichen. Ihr Beschäftigungsrückgang ist deutlich ausgeprägter als in Betrieben, die stärker vorausschauend ein Bündnis zur Verbesserung der Wettbewerbsfähigkeit vereinbaren. Die Initiative zu letzteren Bündnissen geht eher von der Geschäftsleitung aus. Sie sind erfolgreicher als Krisenbündnisse. Insgesamt können Bündnisse zumindest kurz- und mittelfristig bisher nicht als erfolgreich bei der Stabilisierung der Beschäftigung eingestuft werden, obwohl sich durchaus bestimmte Maßnahmen identifizieren lassen, von denen weniger negative oder sogar positive Wirkungen ausgehen.

Festzuhalten ist, dass sich die Untersuchungsergebnisse auf den Zeitraum vor der schweren Rezession des Jahres 2008 beziehen. Dabei zeigte sich, dass Arbeitnehmer in tarifvertraglich gebundenen Betrieben sowohl höhere Löhne erhalten als auch von einer höheren Arbeitsplatzsicherheit profitieren. In den ersten Jahren des neuen Jahrhunderts ist der Flächentarifvertrag weiter erodiert und der Anteil der Betriebe mit einem Betriebsrat weiter gesunken. Nur für Betriebsräte in tarifgebundenen Betriebe konnte noch ein positiver Einfluss auf die Entlohnung nachgewiesen werden, der sich in der zweiten Zeitperiode (2004/2005) nach der Reform des Betriebsverfassungsgesetzes verstärkt hat. Für den betrachteten Zeitraum konnte bei betrieblichen Bündnissen bisher kein allgemeiner beschäftigungsstabilisierender Effekt festgestellt werden.

\section{Stabilität und Entlohnung geförderter Beschäftigung}

Die Wirksamkeit arbeitsmarktpolitischer Maßnahmen und Programme steht in den letzten Jahren verstärkt auf dem Prüfstand. Eingliederungszuschüsse sind zeitlich befristete Lohnkostenzuschüsse, die Arbeitgeber in Deutschland bei der Einstellung von Personen mit eingeschränkter Vermittelbarkeit erhalten können (vgl. Ruppe und Stephan 2009). Sie sollen einen Anreiz zur Einstellung von Personen mit Vermittlungshemmnissen schaffen, indem sie tatsächliche oder vermutete Produktivitätsnachteile der Geförderten ausgleichen. Gegenwärtig beträgt der Förderhöchstsatz 50 Prozent des berücksichtigungsfähigen Arbeitsentgelts und die Förderhöchstdauer zwölf Monaten (für ältere und behinderte Personen gelten Sonderregeln). Wenn ein Unternehmen einen geförderten Arbeitnehmer während der Förderung oder der sogenannten Nachbeschäftigungszeit (deren
Länge der Förderdauer entspricht) kündigt, ohne dass hierfür dringende betriebliche Erfordernisse vorliegen, kann es zur teilweisen Rückzahlung der Zuschüsse verpflichtet werden. Während der Jahre 2007 bis 2009 wurden jeweils etwa 250.000 neu aufgenommene Beschäftigungsverhältnisse mit einem solchen Eingliederungszuschuss gefördert.

Für Deutschland lagen vor Beginn unseres DFG-Projekts keine Befunde zur Stabilität und Entlohnung geförderter Beschäftigung vor. Datengrundlage der Beiträge sind Auszüge aus dem TrEffeR-Datensatz der Bundesagentur für Arbeit (Stephan et al. 2006), zu denen Angaben aus der Beschäftigtenhistorik und der Betriebsdatei des IAB zugespielt wurden. Da in den verwendeten Daten keine detaillierten Informationen zur Arbeitszeit vorliegen, beschränken sich die Auswertungen auf Personen, die eine Vollzeitbeschäftigung aufgenommen haben. International am ehesten vergleichbar ist die Studie von Hamersma (2008), die ein Lohnsubventionsprogramm in Wisconsin untersucht. Sie kommt zu dem Ergebnis, dass die Förderung keine signifikanten Effekte auf die Betriebszugehörigkeitsdauer der Geförderten hatte. Allerdings stellt sie für den Förderzeitraum signifikant positive Effekte auf die Löhne der Teilnehmer fest. Dieses interpretiert sie dahingehend, dass Teile der Förderung - etwa 40 Prozent - in Form einer Lohnprämie an die Arbeitnehmer weitergegeben werden.

Die im Folgenden vorgestellten Arbeiten vergleichen Personen, die ein gefördertes oder ungefördertes Beschäftigungsverhältnis aufgenommen haben. Allein die Tatsache, dass bestimmte Arbeitslose ,nur“ gefördert eingestellt wurden, andere Arbeitslose hingegen auch ohne Lohnkostenzuschuss einen Job finden konnten, weist darauf hin, dass sich beide Personengruppen unterscheiden. Die Studien kontrollieren für eine Vielzahl individueller, betrieblicher und regionaler Merkmale. Stephan (2010a, 2010b) berücksichtigt zudem frühere Löhne, die als Indikator für die individuelle Produktivität herangezogen werden können. Auf Basis der verwendeten Methoden lässt sich jedoch nicht ausschließen, dass zeitvariierende unbeobachtbare Heterogenitäten zwischen gefördert und ungefördert eingestellten Personen verbleiben. Auch könnten sich die Arbeitszeiten der verglichenen Personengruppen - trotz der Beschränkung auf Vollzeitbeschäftigte - im Mittel unterscheiden.

Für Westdeutschland prüft Ruppe (2009), ob sich die Betriebszugehörigkeitsdauer von Personen, die aus Arbeitslosigkeit heraus eine mit einem Eingliederungszuschuss geförderte Vollzeitbeschäftigung fanden, von der ungeförderter Personen unterscheidet. Nach einem Jahr bestand noch etwa die Hälfte der anfänglich geförderten, aber nur ein Drittel der ungefördert aufgenommenen Arbeitsverhältnisse. Auch bei Kontrolle für individuelle, betriebliche und regionale Einflussfaktoren verblieben geförderte Personen signifikant länger im Betrieb. Das Risiko, ein Beschäftigungsverhältnis zu beenden, fällt insbesondere während der För- 
derung als auch während der gesetzlich geregelten Nachbeschäftigungsfrist geringer aus.

Die Einkommens- und Beschäftigungsentwicklung der gleichen Gruppe untersucht Stephan (2010a). Danach unterscheiden sich die Tagesentgelte gefördert und ungefördert eingestellter Personen kurzfristig nicht. Erwähnenswert ist jedoch, dass die Tageslöhne geförderter Männer im Mittel nur leicht über der Niedriglohnschwelle von zwei Drittel des Medianlohnes, bei Frauen jedoch darunter lagen. Da anfänglich geförderte Arbeitnehmer im Folgezeitraum signifikant mehr Tage in Beschäftigung waren, fielen auch ihre kumulierten Verdienste (die sich als das Produkt aus Tagen in Beschäftigung und dem Tageslohn ergeben) höher aus. Förderzeitraum und Nachbeschäftigungsfrist reichen offensichtlich bei vergleichsweise vielen anfänglich geförderten Personen aus, um die Probezeit zu überstehen und ihre Produktivität zu steigern oder Vorbehalte gegenüber ihrer Arbeitsleistung zu entkräften. Aggregiert über den Beobachtungszeitraum von $3 \frac{1}{2}$ Jahren betrugen die zusätzlichen kumulierten Verdienste etwa 2.200 (Frauen in Westdeutschland) bis 4.900 Euro (Männer in Ostdeutschland).

Eine ergänzende Analyse der zehn Branchen, in denen im Beobachtungszeitraum die meisten Eingliederungszuschüsse eingesetzt wurden, zeigt, dass Zuschüsse im 2. Quartal 2003 zahlenmäßig am häufigsten im Bausektor, bei den weiteren wirtschaftlichen Dienstleistungen, im Einzelhandel sowie in der Zeitarbeitsbranche eingesetzt wurden (Stephan 2010b). Die Ergebnisse bestätigen für die meisten Branchen, dass geförderte Arbeitnehmer bei der Einstellung ähnlich hohe Tagesentgelte wie ihre ungefördert eingestellten Kollegen erhielten, dass die Beschäftigungsverhältnisse aber länger andauerten. Zudem wurden geförderte Arbeitnehmer in jeder Branche mit ähnlichen Personen verglichen, die in einem der anderen Sektoren eine geförderte Beschäftigung aufgenommen hatten. Unterschiede zeigten sich bei den Tagesentgelten bei Einstellung, der Beschäftigung wie auch der Betriebszugehörigkeitsdauer, jedoch nicht bei den über $3 \frac{1}{2}$ Jahre kumulierten Verdiensten.

Als Zwischenfazit lässt sich festhalten: Die Tageslöhne geförderter und ähnlicher, aber ungeförderter vormals Arbeitsloser unterscheiden sich kaum. Allerdings beschäftigten Betriebe anfänglich mit einem Eingliederungszuschuss geförderte Personen im Mittel länger als ungefördert eingestellte Arbeitnehmer. Einschränkend ist anzumerken, dass die den Untersuchungen zugrunde liegenden Ansätze keinen Aufschluss über mögliche Mitnahme-, Substitutionsund Verdrängungseffekte der Förderung geben können. Ein Mitnahmeeffekt liegt vor, wenn ein Betrieb genau die Person gefördert einstellt, für die er sich auch ohne eine Förderung entschieden hätte. Ersetzen geförderte Beschäftigte innerhalb eines Betriebes oder in anderen Betrieben ungeförderte Arbeitnehmer, so liegen Substitutions- oder Verdrängungseffekte vor. Der hier durchgeführte Vergleich schätzt den Effekt der Lohnsubvention auf die Entlohnung und die Beschäftigungsdauer der Geförderten unter der Bedingung, dass ein Beschäftigungsverhältnis aufgenommen wurde. Mögliche zusätzliche Effekte der Förderung auf die Aufnahme einer Beschäftigung werden damit ignoriert.

\section{Wirtschaftspolitische Folgerungen}

Aus den empirischen Erkenntnissen der Studien im Rahmen des DFG-Projekts „Beschäftigung zwischen Mobilität und Stabilität" lassen sich verschiedene wirtschaftspolitische Folgerungen ableiten. Dabei geht es nicht nur um staatliche Maßnahmen zur Eingliederung auf dem Arbeitsmarkt und zur besseren Anpassung der Beschäftigung an veränderte Arbeitsmarktbedingungen, sondern auch um Hinweise für Betriebe und Arbeitgeberorganisationen sowie für Betriebsräte, Gewerkschaften und Beschäftigte. Die zentralen Befunde und Folgerungen sind im Folgenden in fünf Absätzen zusammengefasst.

1. Betriebe und Beschäftigte reagieren infolge ihrer Heterogenität sehr unterschiedlich auf staatliche Maßnahmen und institutionelle Vorgaben. Die technologische und organisatorische Struktur der Betriebe bestimmt ganz wesentlich, wie Betriebe auf veränderte Rahmenbedingungen des Arbeitsmarkts reagieren. Betriebe können durch ihre Verhaltensweisen politisch intendierte Wirkungen verstärken oder ihnen entgegen wirken - z.B. durch Reorganisationsmaßnahmen, durch Veränderung des Lohnbildungsprozesses, durch Technologieveränderungen, durch verstärkte Lohnrigidität oder - flexibilität sowie durch Veränderung der Beschäftigungsstruktur.

2. Beschäftigte passen ihr Mobilitätsverhalten an gesamtwirtschaftliche Entwicklungen an. Erwarten sie durch einen Wechsel bessere Arbeitsbedingungen und eine höheren Arbeitszufriedenheit, so nehmen sie auch Einkommensverluste in Kauf. Verfolgen Betriebe das Ziel einer Stabilisierung ihrer Belegschaft, so sollte die betriebliche Personalpolitik vor allem auf eine Verbesserung des Verhältnisses der Kollegen untereinander sowie zu den Vorgesetzten abzielen, die Sicherheit der Arbeitsplätze erhöhen sowie abwechslungsreiche Tätigkeiten anbieten.

3. Rigide Löhne und eine Tarifbindung von Betrieben gehen - dies belegen die Projektarbeiten - mit vorteilhaften Entlohnungs- und Beschäftigungskonstellationen für die Belegschaft einher. Da sich die wirtschaftliche Entwicklung in einer Marktwirtschaft in zyklischen Schwankungen vollzieht, ist es unter Gerechtigkeits- und Teilhabegesichtspunkten erstrebenswert, die Anpassungslasten, die in Rezessionen auftreten, so zu verteilen, dass sie sich nicht überwiegend auf einzelne Gruppen konzentrieren. Auf diese Entwicklung weist auch der Sachverständigenrat zur Begutachtung der gesamtwirtschaft- 
lichen Entwicklung hin (2009, Ziff. 445). In seinem Gutachten (2010, Ziff. 457) betont der Sachverständigenrat jedoch auch, dass ,,die gemeinsamen Anstrengungen aller beteiligten Akteure" erheblich zur robusten Verfassung des Arbeitsmarktes in der Krise beigetragen haben. Aufgeführt werden die Einkommenseinbußen von Arbeitnehmer durch Kurzarbeit, die Übernahme von Kosten bei Kurzarbeit durch Unternehmen, Konjunkturpakete und Ausweitung der Kurzarbeit durch die Bundesregierung, die moderate Lohnpolitik der Gewerkschaften sowie die Weiterbildungsmaßnahmen, auch in Verbindung mit Kurzarbeit, der Bundesagentur für Arbeit (Hübler 2010).

4. Betriebliche Bündnisse, die zwischen dem Arbeitgeber sowie Arbeitnehmern und Betriebsräten vereinbart werden und auf tariflichen Öffnungsklauseln beruhen, sind überwiegend in größeren Betrieben zu finden. Diese Betriebe kombinieren die mit Tarifverträgen verbundene Friedenspflicht mit einer institutionalisierten innerbetrieblichen Kooperation. Die Bündnisse sollen damit einen höheren Grad an Flexibilität ermöglichen. Nach dem gegenwärtigen Kenntnisstand sind betriebliche Bündnisse kurz- und mittelfristig jedoch nicht allzu erfolgreich, was die Beschäftigungswirkungen betrifft. Voraussetzung für einen Erfolg ist, dass Geschäftsleitung und Betriebsrat vertrauensvoll zusammenarbeiten, dass die Bündnisse langfristig angelegt sind, dass sie vor einer wirklichen Unternehmenskrise in Kraft treten und dass die ergriffenen Einzelmaßnahmen aufeinander abgestimmt sind (Hübler 2005a, 2006).

5. Eingliederungszuschüsse sind in Deutschland ein wichtiges Instrument der aktiven Arbeitsmarktpolitik, so dass die Effekte der Förderung auf die Arbeitsmarktchancen und Löhne der Geförderten für Politik und Arbeitsverwaltung von hohem Interesse sind. Die Projektergebnisse zeigen, dass anfänglich geförderte Arbeitnehmer im Mittel pro Tag zunächst etwa genauso viel verdienen wie ähnliche Personen, die ungefördert einen Job aufgenommen haben. Da die Geförderten im Folgezeitraum länger im Betrieb verbleiben und insgesamt höhere Beschäftigungsquoten aufweisen als die Vergleichsgruppe, fallen auch ihre kumulierten Verdienste höher aus. Ergänzende fiskalische Kosten-Nutzen-Analysen weisen darauf hin, dass sich die Förderung hierdurch längerfristig selbst finanzieren kann. Dennoch ist der Einsatz von Eingliederungszuschüssen sorgfältig zu gestalten und zu überwachen: Erstens könnten sich die Wirkungen der Förderung verändern, wenn diese deutlich ausgeweitet wird. Zweitens implizieren positive Wirkungen für die Geförderten noch nicht, dass Eingliederungszuschüsse die Arbeitslosigkeit insgesamt senken. Schließlich ist die Frage von Mitnahmeeffekten - ein geförderter Arbeitnehmer wäre auch ohne Förderung eingestellt worden - derzeit noch nicht hinreichend geklärt.
Ist die wirtschaftspolitische Zielsetzung mehr Sicherheit in Verbindung mit mehr Flexibilität, so können sich Erfolge nur einstellen, wenn die Wirtschaftsakteure in konzertierter Aktion handeln und weniger globale, als vielmehr gruppenspezifische Maßnahmen ergriffen werden. Die vergangene Wirtschaftskrise hat gezeigt, dass es gelingen kann, Beschäftigung auch in der Krise zu sichern. Dies war möglich durch eine Reihe von Sonderfaktoren (betroffen waren vor allem Betriebe in wirtschaftlich an sich starken Regionen), aber auch durch das Zusammenwirken von Belegschaften, Unternehmensleitungen und der Wirtschafts- bzw. Arbeitsmarktpolitik.

\section{Executive summary}

Some consequences for economic policy can be derived from the empirical research results of the DFG-Project "Employment between Mobility and Stability". They are relevant for public activities to support the integration of individuals into the labor market and to facilitate the adaptation of employment to changing labor market conditions, and they are relevant for companies and employers' associations as well as for works councils, unions and employees.

1. A noticeable and in the DFG project well-documented point is the finding that - due to their heterogeneity companies and employees react very differently to public activities and institutional changes. The technological and organizational structure of companies determines to a large extent how they respond to changes of labor market regulations and interventions. The actions of firms can reinforce or counteract politically intended effects, for example by organizational restructuring, modification of the wage-setting regime, technological change, an intensified wage-rigidity or additional wage flexibility as well as by a change of the employment structure.

2. The analyses of the project demonstrate that employees react to macroeconomic developments and adapt their mobility behavior and pattern. Specifically, the expectation of improved working conditions and job satisfaction stimulates mobility to other firms, even accepting a lower remuneration. If companies pursue the goal of stabilizing their work force, their personnel policy should improve the relations between colleagues and supervisors and offer diversified types of activities.

3. Strong hints were found that rigid wages and coverage by a collective agreement contribute to beneficial packages of wages and employment for workers. As economic development in a market economy is subject to cyclical changes, it would be desirable from a perspective of fairness to distribute the burden of adjustment, arising in recessions, in a way that they are not shouldered by 
specific groups. The German Council of Economic Advisors $(2009,2010)$ points out that the joint efforts of all involved parties have significantly contributed to the robust performance of the German labor market during the economic crisis. Workers have accepted income losses through short-time work, firms have taken over part of the costs of short-time work, the government has funded business support programs and short-time work, and unions have negotiated moderate collective contracts (Hübler 2010).

4. Job alliances, contracted by management and works councils and based on opening clauses in collective agreements, are predominantly introduced in larger companies. These firms combine the peace obligations (no strikes) associated with collective contracts and an institutionalized in-plant cooperation of management and works councils. Job alliances are expected to provide a higher degree of flexibility compared with the regulations of collective contracts. The research results show that in the short and medium term job alliances are not successful in stabilizing employment. Prerequisites for a success - employment development is less negative - are that management and works councils collaborate trustfully, job alliances are conceived for a longer period and prior to an imminent crisis and that the measures are coordinated (Hübler 2005a, 2006).

5. Wage subsidies are an important instrument of German labor market policies, thus evaluation results are of interest to labor market politicians and administrators. We find no significant differences in daily wages between subsidized and similar unsubsidized workers, but (initially) subsidized employment relationships are of on average longer duration than similar unsubsidized ones. Furthermore, initially subsidized workers subsequently have generally higher employment rates that result in significantly higher cumulated wages during the time frame investigated. Additional fiscal cost-benefit-analyses suggest that subsidization might thus be self-financing in the longer run. All in all, the results imply that wage subsides have the potential to integrate hard-to-place individuals into the labor market for a longer period than could have been expected without subsidization. Nonetheless, the use of subsidies has to be carefully arranged and supervised: First, the impact of the instrument could diminish if the scale of the program would be extended. Second, positive direct effects on treated persons do not imply that subsidies reduce overall unemployment. Third, the knowledge on the size of deadweight effects - a subsidized worker would have been hired without the help of a subsidy, too - is still scarce.

To conclude, if economic policy strives after more job security combined with more flexibility, successful approaches require consensual actions of all economic actors and group-specific rather than all-encompassing measures. For Germany, the recent demand shock that occurred in the aftermath of the financial crisis has shown that employment may be stabilized also in economically difficult times. Admittedly, this was due to some special features (mainly firms in economically strong regions were affected), but the cooperation of firms' managements and workforces as well as economic and labor market policies played an important role, too.

Danksagung Die Autoren danken der DFG für die Förderung des Projektes „Beschäftigung zwischen Mobilität und Stabilität“ (im Rahmen des Schwerpunktprogramms „Flexibilisierungspotenziale bei heterogenen Arbeitsmärkten“), Thomas Cornelißen, Kathi Ruppe und Stefan Schneck für die gute Zusammenarbeit im Projekt sowie einem anonymen Gutachter und Bernd Fitzenberger als Herausgeber für wertvolle Hinweise.

\section{Literatur}

Altonji, J.G., Devereux, P.J.: The extent and consequences of downward nominal wage rigidity. Res. Labor Econ. 19, 383-431 (2000)

Beckmann, M., Cornelißen, T., Schauenberg, B.: Fixed-term employment, work organization and job satisfaction: evidence from German individual-level data. WWZ discussion paper 08/09, Universität Basel (2009)

Bellmann, L., Cornelißen, T., Hübler, O., Pahnke, A.: Betriebliche Reorganisation, Entlohnung, Beschäftigungsstabilität. Z. ArbeitsmarktForsch. 41, 259-285 (2008a)

Bellmann, L., Gerlach, K., Meyer, W.: Company-level pacts for employment. Jahrb. Natl.ökon. Stat. 228, 533-553 (2008b)

Card, D., de la Rica, S.: The effect of firm-level contracts on the structure of wages: evidence from matched employer-employee data. Ind. Labor Relat. Rev. 59, 573-592 (2006)

Cornelißen, T.: The interaction of job satisfaction, job search, and job changes. An empirical investigation with German panel data. J. Happiness Stud. 10, 367-384 (2009)

Cornelißen, T., Hübler, O.: Downward wage rigidity and job mobility. Empir. Econ. 34, 205-230 (2008)

Cornelißen, T., Hübler, O.: Unobserved individual and firm heterogeneity in wage and job-duration functions: evidence from German linked employer-employee data. German Economic Review (2011, forthcoming). doi:10.1111/j.1468-0475.2010.00528.x.

Farber, H.S.: Mobility and stability: the dynamics of job change in labor markets. In: Ashenfelter, O., Card, D. (Hrsg.) Handbook of Labor Economics, Bd. 3B, S. 2439-2483. Elsevier, Amsterdam (1999)

Gerlach, K., Hübler, O., Meyer, W.: The Hannover firm panel (HFP). Schmollers Jahrb. Wirtsch.- Soz.wiss. 123, 463-470 (2003)

Gerlach, K., Hübler, O.: Employment adjustment on the internal and external labour market-an empirical study with personnel records of a German company. Jahrb. Natl.ökon. Stat. 229, 198-213 (2009)

Gerlach, K., Meyer, W.: Wage effects of works councils and collective agreements in Germany. In: Brown, C., Eichengreen, B., Reich, M. (Hrsg.) Labor in the Era of Globalization, S. 295-316. Cambridge University Press, Cambridge (2010)

Gerlach, K., Stephan, G.: A note on individual tenure and collective contracts. Labour 22, 167-183 (2008)

Gerlach, K., Stephan, G.: Wage settlements and wage setting: results from a multi-level model. Appl. Econ. 37, 2297-2306 (2005) 
Hamersma, S.: The effects of an employer subsidy on employment outcomes: a study of the work opportunity and welfare-to-work tax credits. J. Policy Anal. Manage. 27, 498-520 (2008)

Hübler, O.: Labor hoarding and short-time work in Germanycomment. Appl. Econ. Q. Suppl. 61, 151-165 (2010)

Hübler, O.: Sind betriebliche Bündnisse erfolgreich? Jahrb. Natl.ökon. Stat. 225, 630-652 (2005a)

Hübler, O.: Betriebliche Vereinbarungen zur Beschäftigungs- und Standortsicherung. In: L. Bellmann, O. Hübler, W. Meyer, G. Stephan (Hrsg.) Institutionen, Löhne und Beschäftigung, BeitrAB 294, Nürnberg, S. 157-173 (2005b)

Hübler, O.: Zum Einfluss betrieblicher Bündnisse auf die wirtschaftliche Lage der Unternehmen. Jahrb. Wirtsch.wiss. 57, 121-146 (2006)

Hübler, D., Hübler, O.: The link between job security and wages: a comparison between Germany and the UK. Schmalenbach Bus. Rev. 62, 45-67 (2010)

Hübler, O., Jirjahn, U.: Works councils and collective bargaining in Germany: the impact on productivity and wages. Scott. J. Polit. Econ. 50, 471-491 (2003)

Hübler, O., Walter, T.: Beschäftigungsstabilität im Konjunkturzyklus - Eine empirische Analyse mit Individualdaten für Westdeutschland 1984-2005. Schmollers Jahrb. Wirtsch.- Soz.wiss. 129, 1-35 (2009)

Pfeifer, C., Schneck, S.: (2010), Relative wage positions and quit behavior: new evidence from linked employer-employee data. Discussion Paper No. 438, Leibniz Universität Hannover

Pfeiffer, F.: Lohnrigiditäten im gemischten Lohnbildungssystem, ZEW Wirtschaftsanalysen, Bd. 65. Nomos, Baden-Baden (2003)

Ruppe, K.: Eingliederungszuschüsse und Betriebszugehörigkeit in Westdeutschland. IAB discussion paper 6/2009 (2009)

Ruppe, K., Stephan, G.: Förderung mit Eingliederungszuschüssen: Länger im Betrieb und gleicher Lohn. IAB-Kurzbericht 25/2009 (2009)

Sachverständigenrat zur Begutachtung der gesamtwirtschaftlichen Entwicklung: Jahresgutachten 2009/10. Die Zukunft nicht aufs Spiel setzen (www.sachverstaendigenrat.de/jahresgutachten-2009 -2010.html) (2009)

Sachverständigenrat zur Begutachtung der gesamtwirtschaftlichen Entwicklung: Jahresgutachten 2010/11. Chancen für einen stabilen Aufschwung (www.sachverstaendigenrat.de/ aktuellesjahresgutachten-2010-2011.html) (2010)

Schneck, S.: Why mobile workers voluntarily change to lower wages. Mimeo (2010)

Stephan, G., Rässler, S., Schewe, T.: Das TrEffeR-Projekt der Bundesagentur für Arbeit: Die Wirkung von Maßnahmen aktiver Arbeitsmarktpolitik. Z. ArbeitsmarktForsch. 39, 447-466 (2006)
Stephan, G.: Employer wage subsidies and wages in Germany: empirical evidence from individual data. Z. ArbeitsmarktForsch. 43, 53-71 (2010a)

Stephan, G.: Wages, employment and tenure of temporarily subsidized workers: does the industry matter? IAB discussion paper 12/2010 (2010b)

Knut Gerlach 1960-1965 Studium der Volkswirtschaftslehre an den Universitäten Göttingen und Montpellier. 1965 Abschluss als DiplomVolkswirt an der Universität Göttingen. 1969 Promotion an der Universität Göttingen. 1967-1974 wissenschaftlicher Mitarbeiter an der Universität Regensburg. 1974 Habilitation in Regensburg. 1974-2005 Professor für Arbeitsökonomie an der Universität Hannover. Seit 2005 Professor emeritus. Mehrere Forschungsfreisemester und Studienaufenthalte an der University of California, Berkeley.

Forschungsfelder: Empirische Arbeitsmarktforschung, Tarifbindung und Entlohnung, betriebsinterne Arbeitsmärkte.

Olaf Hübler Studium der Volkswirtschaftslehre an der FU Berlin, 1970 Abschluss als Diplom-Volkswirt. Von 1970-1975 wissenschaftlicher Mitarbeiter am Lehrstuhl für Finanzwissenschaft und im Assistententeam für das volkswirtschaftliche Grundstudium an der TU Berlin. 1974 Promotion zum Dr. rer. pol., 1978 Habilitation. Seit 1982 Professor für Ökonometrie an der Universität Hannover. Research Fellow am Forschungsinstitut zur Zukunft der Arbeit (IZA) in Bonn seit 1999 und Research Fellow am Institut für Arbeitsmarkt- und Berufsforschung (IAB) in Nürnberg seit 2005. Von 2006-2008 Sprecher des DFG-Schwerpunktprogramms ,Flexibilisierungspotenziale bei heterogenen Arbeitsmärkten“. Associate Editor von „Advances in Statistical Analysis (AStA)“.

Forschungsfelder: Empirische Wirtschaftsforschung, Mikroökonometrie, Arbeitsmarktforschung.

Gesine Stephan Head of the Research Unit „Active Labour Market Policies and Integration" (since April 2004) and Professor of Economics (Volkswirtschaftslehre), in particular empirical microeconomics, at the Friedrich Alexander University of Erlangen-Nuremberg (since May 2009). She studied economics at the University of Hannover from 1984 to 1990 , where she also received her doctoral degree in political science in 1994 and completed her habilitation in 2000.

Research fields: Applied micro-econometric labour market research. The results of her work have been published in Applied Economics, the Cambridge Journal of Economics, Economics Letters, Industrial Relations, and other journals. 